\title{
Combating antimicrobial resistance during the COVID-19 pandemic
}

\author{
Edmond SK Ma ${ }^{1,2}$ *, FHKAM (Community Medicine), KH Kung22, MMedSc, FHKAM (Community Medicine), \\ Hong Chen², MPH, FHKAM (Community Medicine) \\ ${ }^{1}$ Epidemiology Adviser, Hong Kong Medical Journal \\ ${ }^{2}$ Infection Control Branch, Centre for Health Protection, Department of Health, Hong Kong SAR Government, Hong Kong \\ *Corresponding author: edmond_sk_ma@dh.gov.hk
}

Hong Kong Med J 2021;27:396-8

https://doi.org/10.12809/hkmj215124

Global attention has been drawn to combat coronavirus disease 2019 (COVID-19), draining tremendous government resources, political commitment, public health measures, manpower of healthcare professionals, media, and public interest. The COVID-19 has also seriously affected the global effort against antimicrobial resistance (AMR). According to a survey conducted in late 2020 by the World Health Organization (WHO) AMR Surveillance and Quality Assessment Collaborating Centres Network, 63\% (35 out of 56 countries) reported an increase in total prescriptions of antibiotics, with $47 \%(23 / 49), 57 \%(27 / 47)$, and $40 \%$ $(18 / 45)$ of countries reporting increased use of WHO Access, Watch, and Reserve antibiotics, respectively. ${ }^{1}$ More importantly, 37\% (13/35) and 40\% (12/30) of countries reported an increase in multidrugresistant organism (MDRO) healthcare-associated infections and MDRO infections in long-term care facilities, respectively. Outbreaks and increases in AMR acquisition, such as carbapenemase-producing Enterobacteriaceae, have also been reported by hospitals during the COVID-19 pandemic. ${ }^{2-4}$

The situation of MDRO in Hong Kong is worrisome. The Hospital Authority has reported a higher rate of methicillin-resistant Staphylococcus aureus bacteraemia detected after 48 hours of admission in 2020 and the first half of 2021, compared with that of previous years, although this may be due to a reduction in hospital admission of milder cases. $^{5}$ An outbreak of Candida auris, an often highly resistant emerging infection, during the third wave of the COVID-19 pandemic is also concerning. From 29 June 2020 to 9 October 2020, the Hospital Authority reported 41 patients with Candida auris colonisation to the Infection Control Branch of the Centre for Health Protection for infection control advice after discharge to residential care homes. ${ }^{6}$ The number of cases of carbapenem-resistant Enterobacteriaceae discharged to residential care homes for the elderly rose from 242 cases in 2019 to 259 cases in 2020, and then sharply to 329 cases between January and August 2021.

The COVID-19 pandemic has affected antimicrobial stewardship activities and driven
AMR in various ways. Drifting of resources, including laboratory capacity, reduced reagents and consumables, physician and nursing manpower, and public health staff, have undermined antimicrobial stewardship programmes in many countries. Weakened infection control due to fatigue and heavy workload of healthcare workers, and shortages of personal protective equipment in the early days of the pandemic further aggravated the problem. Hospital admission may increase the risk of healthcare-associated infections and the transmission of MDROs, which in turn may lead to increased antimicrobial use. Disruption to routine immunisation activities, due to COVID-19related measures, has led to reductions in overall vaccination coverage globally, potentially leading to an overuse of antimicrobials. ${ }^{7-9}$ Low-level exposure to biocidal agents can strengthen drug-resistant strains and enhance the risk of cross resistance to antibiotics, particularly those that treat Gramnegative bacteria. ${ }^{10}$

Another important impact of COVID-19 on increasing AMR is secondary bacterial infection among patients with COVID-19. Empirical treatment of patients with COVID-19 using antibiotics is common. A meta-analysis involving 154 studies and over 30000 patients revealed that $74.6 \%$ of patients with COVID-19 received antibiotics, significantly higher than the estimated prevalence of bacterial co-infection. ${ }^{11}$ This is echoed by another review of studies published on hospitalised patients with COVID-19, which revealed $72 \%(1450 / 2010)$ of patients received antibiotics but only $8 \%(62 / 806)$ had bacterial or fungal co-infections. ${ }^{12}$ The most common type of secondary infection of COVID-19 was pneumonia (especially ventilator-associated pneumonia), followed by bloodstream and urinary tract infections, and the most commonly used agents included fluoroquinolones, cephalosporins, carbapenems, azithromycin, vancomycin, and linezolid. ${ }^{13,14}$ Those with complications of COVID-19 may require mechanical ventilation or other invasive devices, which increases the risk of acquiring hospital-associated pathogens that are often highly resistant, such as methicillin-resistant 
Staphylococcus aureus, Pseudomonas aeruginosa, and Acinetobacter baumannii. ${ }^{15}$

Despite the challenges posed to AMR, the COVID-19 pandemic also creates some opportunities, such as enhanced infection control measures among healthcare workers and the high alertness of personal hygiene among the general public. These measures, which include cough etiquette, hand hygiene, wearing of masks, and social distancing, have helped to reduce various infections, in particular those caused by respiratory pathogens. $^{16-18}$ In addition, increased fear of attending healthcare facilities and postponement of elective hospital procedures have resulted in fewer medical consultations and antibiotic prescriptions. Surveillance data on wholesale antimicrobial consumption in Hong Kong show a substantial reduction (28.3\%) in daily defined doses of antimicrobials from 19.02 million in 2019 to 13.63 million in 2020 . In particular, there were major reductions (of between $35.3 \%$ and 51.5\%) in wholesale supply to general practitioners of amoxicillin with or without beta-lactamase inhibitor, azithromycin, and cefuroxime, which are commonly prescribed to manage upper and lower respiratory tract infections. In Hong Kong, social distancing measures imposed by public health authorities, school and kindergarten closures, work-fromhome policies, other restrictions involving catering businesses and scheduled premises such as fitness centres, beauty salons, karaoke establishments and sport centres, have all helped limit transmission through the respiratory route and person-to-person contact of not only COVID-19 but also other infections. Enhanced environmental hygiene using diluted household bleach containing $5.25 \%$ sodium hypochlorite can kill severe acute respiratory syndrome coronavirus 2 and other pathogens including MDROs.

Although more research is needed to dissect the intermingled relationship between COVID-19 and AMR, it is of utmost importance to maintain efforts against AMR. The theme for World Antibiotic Awareness Week, held on 18 to 24 November 2021, was "Spread Awareness, Stop Resistance". The dedicated page on the Centre for Health Protection website (https://www.chp.gov.hk/en/features/47850. html) includes access to the latest IMPACT (Interhospital Multi-disciplinary Programme on Antimicrobial ChemoTherapy) guideline, an evidence-based clinical guideline to ensure that patients receive the right antibiotic, at the right dose, at the right time, and for the right duration that leads to the best clinical outcome for the treatment or prevention of infection while producing low risk for subsequent resistance. The website also allows access to patient education and publicity materials on AMR. Similar to COVID-19, AMR is a complex global priority and everyone has a role to play.

\section{Author contributions}

All authors contributed to the editorial, approved the final version for publication, and take responsibility for its accuracy and integrity.

\section{Conflicts of interest}

The authors have declared no conflict of interest.

\section{Funding/support}

This study received no specific grant from any funding agency in the public, commercial, or not-for-profit sectors.

\section{References}

1. Tomczyk S, Taylor A, Brown A, et al. Impact of the COVID-19 pandemic on the surveillance, prevention and control of antimicrobial resistance: a global survey. J Antimicrob Chemother 2021;76:3045-58.

2. Tiri B, Sensi E, Marsiliani V, et al. Antimicrobial Stewardship Program, COVID-19, and infection control: spread of carbapenem-resistant Klebsiella pneumoniae colonization in ICU COVID-19 patients. What did not work? J Clin Med 2020;9:2744.

3. Farfour E, Lecuru M, Dortet L, et al. Carbapenemaseproducing Enterobacterales outbreak: another dark side of COVID-19. Am J Infect Control 2020;48:1533-6.

4. Gomez-Simmonds A, Annavajhala MK, McConville TH, et al. Carbapenemase-producing Enterobacterales causing secondary infections during the COVID-19 crisis at a New York City hospital. J Antimicrob Chemother 2021;76:3804.

5. Hospital Authority, Hong Kong SAR Government. MRSA bacteremia in HA Hospitals-2021-Q1 \& 2021-Q2. Available from: https://www.ha.org.hk/haho/ho/cico/ MRSA_Bacteremia_in_HA_hospitals_2021Q1_2021Q2. pdf. Accessed 12 Sep 2021.

6. Centre for Health Protection, Department of Health, Hong Kong SAR Government. Letter to doctor: alert on the rise in Candida auris colonisation in Hong Kong. Available from: https://www.chp.gov.hk/files/pdf/lti_c_auris_20201015_ eng.pdf. Accessed 12 Sep 2021.

7. Bramer CA, Kimmins LM, Swanson R, et al. Decline in child vaccination coverage during the COVID-19 pandemicMichigan Care Improvement Registry, May 2016-May 2020. MMWR Morb Mortal Wkly Rep 2020;69:630-1.

8. McDonald HI, Tessier E, White JM, et al. Early impact of the coronavirus disease (COVID-19) pandemic and physical distancing measures on routine childhood vaccinations in England, January to April 2020. Euro Surveill 2020;25:2000848.

9. Saxena S, Skirrow H, Bedford H. Routine vaccination during covid-19 pandemic response. BMJ 2020;369:m2392. Erratum in: BMJ 2020;369:m2435.

10. Getahun H, Smith I, Trivedi K, Paulin S, Balkhy HH. Tackling antimicrobial resistance in the COVID-19 pandemic. Bull World Health Organ 2020;98:442-442A.

11. Langford BJ, So $M$, Raybardhan $S$, et al. Antibiotic prescribing in patients with COVID-19: rapid review and meta-analysis. Clin Microbiol Infect 2021;27:520-31.

12. Rawson TM, Moore LS, Zhu N, et al. Bacterial and fungal coinfection in individuals with coronavirus: a rapid review 
to support COVID-19 antimicrobial prescribing. Clin Infect Dis 2020;71:2459-68.

13. Clancy CJ, Buehrle DJ, Nguyen MH. PRO: The COVID-19 pandemic will result in increased antimicrobial resistance rates. JAC Antimicrob Resist 2020;2:dlaa049.

14. Clancy CJ, Nguyen MH. Coronavirus disease 2019, superinfections, and antimicrobial development: what can we expect? Clin Infect Dis 2020;71:2736-43.

15. Knight GM, Glover RE, McQuaid CF, et al. Antimicrobial resistance and COVID-19: intersections and implications. Elife 2021;10:e64139.

16. Wan WY, Thoon KC, Loo LH, et al. Trends in respiratory virus infections during the COVID-19 pandemic in Singapore, 2020. JAMA Netw Open 2021;4:e2115973.

17. Park KY, Seo S, Han J, Park JY. Respiratory virus surveillance in Canada during the COVID-19 pandemic: an epidemiological analysis of the effectiveness of pandemic-related public health measures in reducing seasonal respiratory viruses test positivity. PLoS One 2021;16:e0253451.

18. Tanislav C, Kostev K. Fewer non-COVID-19 respiratory tract infections and gastrointestinal infections during the COVID-19 pandemic. J Med Virol 2021 Sep 7. Epub ahead of print. 